Revue Interventions économiques

Papers in Political Economy

56 | 2016

Développement et inclusion. Le grand chantier des inégalités, de la pauvreté

\title{
Martin Ravaillon, The Economics of Poverty: History, Measurement and Policy, Oxford University Press, 2016, $702 \mathrm{p}$.
}

Chalmers LaRose

\section{OpenEdition}

\section{Journals}

Electronic version

URL: http://journals.openedition.org/interventionseconomiques/2946

DOI: 10.4000/interventionseconomiques.2946

ISBN: $1710-7377$

ISSN: $1710-7377$

Publisher

Association d'Économie Politique

Electronic reference

Chalmers LaRose, «Martin Ravaillon, The Economics of Poverty: History, Measurement and Policy, Oxford University Press, 2016, 702 p. », Revue Interventions économiques [Online], 56 | 2016, Online since 18 October 2016, connection on 24 September 2020. URL : http://journals.openedition.org/ interventionseconomiques/2946 ; DOI : https://doi.org/10.4000/interventionseconomiques.2946

This text was automatically generated on 24 September 2020

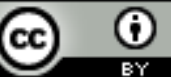

Les contenus de la revue Interventions économiques sont mis à disposition selon les termes de la Licence Creative Commons Attribution 4.0 International. 


\title{
Martin Ravaillon, The Economics of Poverty: History, Measurement and Policy, Oxford University Press, 2016, 702 p.
}

\author{
Chalmers LaRose
}

1 Les sociétés organisées modernes sont jugées à l'aune de la manière dont elles traitent leurs couches les plus vulnérables. Depuis le $16^{\mathrm{e}}$ siècle, période qui a vu l'introduction des Poor Laws (Lois sur les indigents) en Angleterre, la pauvreté - son accroissement aussi bien que les meilleurs moyens de combattre ce phénomène humainement et socialement dégradant - occupe une place centrale dans le débat public.

2 Aujourd'hui il existe une cohorte d'économistes qui, à l'instar des James K. Galbraith, Thomas Piketty, Angus Deaton, et bien d'autres encore, consacrent une très grande partie de leur cheminement professionnel à l'étude du phénomène des inégalités et de la pauvreté dans les sociétés organisées au nord comme au sud. Martin Ravaillon fait figure de proue au sein de ce club illustre. Toutefois la particularité de l'économiste australien est attribuable à son parcours pour le moins singulier. En effet, Ravaillon a passé l'essentiel de sa carrière au sein de la Banque mondiale comme expert des questions relatives à la lutte contre la pauvreté dans le monde. À ce titre, il a observé, pensé et proposé des politiques sur l'ensemble des secteurs d'intervention de l'institution internationale dans ce domaine.

3 Au terme de cette aventure dans le fonctionnariat international, et fort d'un fonds de savoirs et d'expériences accumulés au cours de nombreuses années, l'auteur nous offre un ouvrage testament dans lequel il aborde un ensemble de sujets couvrant les aspects économiques, historiques et sociaux de la pauvreté. Aujourd'hui Martin Ravaillon occupe un poste à l'université Georgetown où il dispense un cours fondamental sur le sujet.

4 The Economics of Poverty: History, Measurement and Policy est une brique de 702 pages. L'ouvrage propose une perspective à la fois historique et globale sur la manière de 
penser la pauvreté. À ce sujet, tant les débats antérieurs et actuels qui interviennent dans les pays riches aussi bien qu'à l'intérieur des pays pauvres y sont abordés avec clarté et profondeur. Par le biais d'une synthèse accessible de la pensée économique, l'auteur apporte également des réponses à certaines questions brûlantes et d'intérêt majeur: Comment la pauvreté est-elle mesurée ? Combien y a-t-il de personnes vivant dans la pauvreté dans le monde? Pourquoi la pauvreté existe? Qu'est-ce qui peut être fait pour la réduire, voire même l'éliminer? Quelles en sont les politiques les plus appropriées et les mieux adaptées?

5 L'ouvrage se décline en trois parties. L'aventure commence par une histoire de la pensée économique dans laquelle l'auteur met en relief les idées mercantilistes sur l'inévitabilité de la pauvreté. Ici Ravaillon explique comment la pauvreté en est venue à être perçue comme un «mal social» capable d'être réduit de manière significative, voire même éliminée. En même temps, il présente les idées qui sous-tendent les politiques anti-pauvreté en apportant un éclairage opportun sur les effets incitatifs qui consistent à aider les personnes pauvres, et le changement d'emphase qui s'y est installé. Dans cette première partie, l'auteur soulève des discussions très animées sur la guerre contre la pauvreté aux États-Unis d'Amérique, de même que les approches initiées en Grande-Bretagne en plus de faire ressortir l'accent mis récemment sur les pays en développement.

6 Les questions de nature conceptuelle et certains problèmes liés aux instruments de mesure de la pauvreté et aux méthodes constituent l'intégralité de la deuxième partie de l'ouvrage. Ici les concepts clés y sont expliqués de façon intuitive, incluant les nombreux débats au sujet de la meilleure manière d'évaluer le bien-être et de mesurer la pauvreté et les inégalités. L'auteur propose des explications prudentes sur un ensemble de questions que soulève la conceptualisation de la pauvreté, notamment la manière dont on établit les seuils de pauvreté, les prix, les échelles subjectives, etc. Ravaillon y aborde également la manière dont a été établi le seuil de pauvreté aux États-Unis, comment la Russie a établi son seuil de pauvreté basée sur des manières différentes de fixer le prix des paniers de consommation lequel varie d'un point à l'autre du territoire.

7 Quant à la troisième partie, elle est en partie prescriptive. Celle-ci est intégralement réservée aux politiques de lutte contre la pauvreté. L'ouvrage fournit un panorama général des tendances en matière de pauvreté à travers le monde, et un tour d'horizon d'un ensemble d'approches différentes en vue de lutter contre la pauvreté, incluant des politiques macro et sectorielles y compris des politiques ciblées et spécifiques. Après avoir passé en revue les savoirs actuels sur les dimensions de la pauvreté et des inégalités dans le monde, le lecteur fait connaissance avec les théories et les données concernant la distribution des bénéfices émanant de la croissance économique aussi bien que les sources de cette croissance. La discussion s'étend aussi aux politiques économiques sectorielles plus larges et aux interventions directement ciblées en direction des personnes pauvres. Ici l'emphase est mise sur l'explication des idées qui sous-tendent les politiques, et des discussions sur les raisons pour lesquelles celles-ci ne fonctionnent pas toujours comme espérée.

8 Somme toute, il s'agit d'un ouvrage qui peut servir indifféremment de manuel pédagogique et de référence de portée générale qui intéressera à la fois les chercheurs aussi bien que les professionnels travaillant pour les gouvernements et les organisations internationales. Bien que l'auteur ne semble pas opter pour une 
politique anti-pauvreté particulière, il aurait été utile toutefois que l'ouvrage rende des discussions au sujet des programmes novateurs, comme les transferts monétaires conditionnés et la manière dont ceux-ci orientent les politiques publiques actuelles au sein des pays en développement. Néanmoins il s'agit d'un ouvrage pertinent lequel conviendrait donc à la fois à un auditoire éclairé composé de lecteurs orientés vers l'élaboration de politiques anti-pauvreté.

9 Au final, l'ouvrage demeure une lecture d'intérêt tant pour ceux qui considèrent enseigner un cours sur la pauvreté que pour ceux-là qui travaillent dans le domaine ou sont à la recherche d'une mise à jour sur certains concepts et questions clés. 